\title{
REDES NEURAIS APLICADAS A RELÉS DIFERENCIAIS PARA TRANSFORMADORES DE POTÊNCIA
}

\author{
Ênio Carlos Segatto* \\ segatto@sel.eesc.usp.br
}

\author{
Denis Vinicius Coury * \\ couryesel.eesc.usp.br
}

\author{
${ }^{*}$ Depto de Engenharia Elétrica \\ EESC - Escola de Engenharia de São Carlos \\ USP - Universidade de São Paulo \\ Av. Trabalhador Sãocarlense, 400 \\ São Carlos - SP - Brasil
}

\section{RESUMO}

Este trabalho apresenta um sistema completo de proteção diferencial para transformadores de potência, através da teoria de Redes Neurais Artificiais (RNAs). O método proposto trata a classificação do sistema de proteção como um problema de reconhecimento de padrões e constitui um método alternativo aos algoritmos convencionais. Muitos fatores, tais como a energização do transformador e a saturação dos TCs, podem causar uma operação inadequada do relé de proteção. Um sistema de proteção completo foi desenvolvido, incluindo um módulo baseado em RNA em substituição aos filtros harmônicos, usados no algoritmo convencional. Este módulo se constituiu de uma RNA tipo MLP Backpropagation para a classificação de sinais. Abordagens baseadas na reconstrução dos sinais distorcidos causados pela saturação dos TCs são também propostas. Essa análise foi realizada através do emprego de RNAs Recorrentes de Elman, utilizadas para reconstruir os sinais distorcidos pela saturação dos TCs. Essas rotinas foram adicionadas ao algoritmo final de proteção. O desempenho dos algoritmos propostos foi comparado ao do algoritmo convencional de proteção de transformadores, em termos de velocidade e precisão de resposta. Com a utilização de uma ferramenta de inteligência artificial em um algoritmo completo de proteção de transformadores,

\footnotetext{
Artigo submetido em 18/04/2007

1a. Revisão em 27/08/2007

2a. Revisão em 12/12/2007

Aceito sob recomendação do Editor Associado Prof. Carlos A. Castro
}

uma solução precisa, rápida e eficiente foi obtida, se comparada aos métodos convencionais.

PALAVRAS-CHAVE: Proteção Diferencial, Redes Neurais Artificiais, Transformadores de Potência, Saturação do Transformador de Corrente.

\section{ABSTRACT}

This paper presents a complete differential protection system for power transformers, applying the Artificial Neural Network (ANN) theory. The proposed approach treat the classification of the protection system as a problem of pattern recognition and as an alternative method to the conventional algorithms. Several factors such as, for example, transformer energization and CT saturation can cause an inadequate operation of the protection relay. A complete protection system was developed, including an ANN-based device in substitution to harmonic filters in use in the conventional algorithm. This stage was carried out by a MLP Backpropagtion ANN to the signals classification. Some approaches concerning the reconstruction of the distorted signals caused by the CTs saturation are also proposed. This analysis was made by Elman recurrent ANNs used to reconstruct the distorted signals caused by CT saturation. These routines are added to the final protection algorithm. With the use of artificial intelligence tools in a complete power transformer protection algorithm, a very precise, fast and efficient solution was obtained, if compared to the conventional methods. 
KEYWORDS: Differential Protection; Artificial Neural Networks; Power Transformers; Current Transformer Saturation.

\section{INTRODUÇÃO}

Transformadores são dispositivos que requerem manutenção especial devido a sua importância ao sistema elétrico no qual eles são conectados. Geralmente, relés diferenciais são usados na proteção primária de grandes transformadores. Em tais relés, as correntes diferenciais são comparadas a um patamar preestabelecido e no caso de uma falta interna, o transformador deverá ser desconectado do resto do sistema. Entretanto, a simples detecção de uma corrente diferencial não é suficiente para distinguir faltas internas de outras situações que também produzem tal corrente. Algumas destas situações surgem durante a energização do transformador (correntes de inrush), saturação do TC (transformador de corrente), entre outras, as quais podem resultar em um trip incorreto (Horowitz e Phadke, 1995).

A correta e rápida distinção de faltas internas de outras situações mencionadas é um dos principais desafios da moderna proteção de transformadores. Com relação à identificação entre faltas internas e correntes de inrush, o método tradicionalmente utilizado é a lógica diferencial com restrição harmônica. Neste método, a corrente de inrush do transformador devido à energização é reconhecida com base nos componentes de segundo harmônico (acima de 16\%) obtidas por filtros. Entretanto, o método de filtragem pode eventualmente atrasar o processo de proteção. Em adição a isso, componentes de segunda harmônica podem também estar presentes durante situações de falta interna (Alternative Transients Program Rule Book, 1987).

Novos métodos com o objetivo de melhorar a seletividade, sensibilidade e a operação de relés diferenciais, tais como redes neurais e lógica Fuzzy, têm sido estudados nos últimos anos. Diferentes arquiteturas são apresentadas na literatura com bons resultados (Yu et alii, 1999; Yu et alii, 2001; Kang et alii, 1996; Kang et alii, 1997; Jamali e Radji, 1997; Sidhu et alii, 1999). A maioria delas utiliza a rede Multi-Layer Perceptron (MLP) e o método Backpropagation.

Este trabalho apresenta um método alternativo utilizando Redes Neurais Artificiais (RNAs) com o objetivo de melhorar o desempenho da proteção de transformadores de potência com relação à correta identificação de faltas internas e correntes de inrush, como descrito anteriormente. Várias arquiteturas foram avaliadas, bem como métodos de treinamento distintos, e o trabalho mostra as melhores configurações de RNAs obtidas. A rede neural MLP com o método Backpropagation foi a arquitetura escolhida na tarefa de classificação de sinais.
Neste artigo, o programa ATP (Alternative Transients Program) foi usado para modelar o transformador de potência no sistema elétrico. Esse modelo foi ainda usado para a geração de dados saturados e não saturados para o treinamento das RNAs recorrentes, responsáveis pela etapa de reconstrução de sinais saturados, bem como para os testes das abordagens propostas. Resultados encorajadores relacionados à nova metodologia são apresentados na última seção.

\section{A PROTEÇÃO DIFERENCIAL}

O diagrama ilustrando a lógica diferencial usada na proteção de grandes transformadores de potência é mostrado na Figura 1. A ilustração também mostra a conexão dos transformadores de corrente (TCs) acoplados aos ramos primário e secundário. O valor N1:N2 é a relação de espiras entre os enrolamentos primário e secundário do transformador, e 1: $\mathrm{n}_{1}$ e 1:n $\mathrm{n}_{2}$ são as relações de espiras entre os ramos e os TCs, selecionados para fazer $\mathrm{N} 1 \mathrm{n}_{1}=\mathrm{N} 2 \mathrm{n}_{2}$. Sob condições normais e de faltas externas a um transformador monofásico, as correntes $\mathrm{i}_{1 S}$ e $\mathrm{i}_{2 S}$ (correntes secundárias dos TCs) são iguais. Porém, no caso de faltas internas, a diferença entre estas correntes torna-se significativa, causando a atuação do relé de sobrecorrente.

Portanto, a corrente diferencial

$$
i_{d}=i_{1 S}-i_{2 S}
$$

fornece uma medida precisa da corrente de falta.

A fim de corrigir erros introduzidos pelos TCs e pelas mudanças de tap do transformador, um patamar é fixado para que uma pequena corrente diferencial possa ser tolerada, sem desconectar o sistema. Essa relação é mostrada na equação 2.

$$
i_{d} \geq K\left(i_{1 S}+i_{2 S}\right) / 2
$$

onde K é a inclinação da característica diferencial.

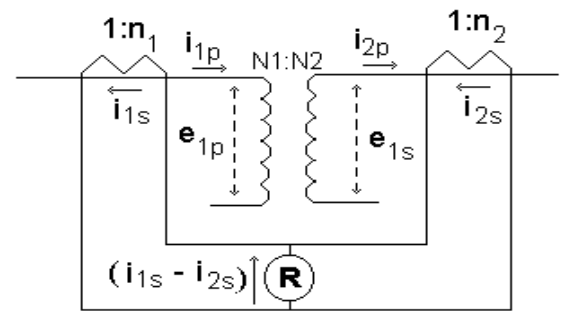

Figura 1: Esquema diferencial usado na proteção de grandes transformadores de potência 


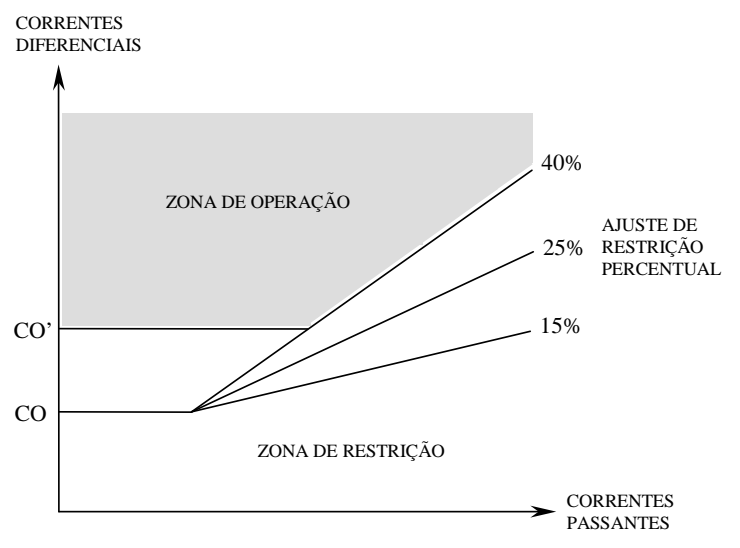

Figura 2: Curva característica diferencial

A Figura 2 mostra a característica diferencial do relé incluindo as zonas de operação e restrição. São apresentados alguns ajustes para K $(15 \%, 25 \%$ e $40 \%)$. CO' é um patamar usado quando uma condição de sobreexcitação é detectada.

Como já citado, certos fenômenos podem causar uma corrente diferencial considerável, mesmo sem existir uma situação de falta e estes falsos sinais são geralmente suficientes para causar o acionamento do relé. Entretanto, nestes casos, a proteção diferencial não deveria desconectar o sistema porque uma falta interna não estaria caracterizada.

Correntes de magnetização (inrush) surgem durante a energização do transformador devido à magnetização e saturação de seu núcleo. A declividade da característica de magnetização na área saturada determina sua magnitude. Em transformadores modernos, altas correntes de inrush podem surgir (Horowitz e Phadke, 1995). Como o lado secundário está aberto na energização do transformador, a corrente diferencial pode alcançar valores suficientemente altos, originando uma operação indevida do relé. A modelagem de tal situação tem sido estudada, mostrando a predominância do componente de $2^{a}$ harmônica. (Cummins et alii, 2000; Bozoki e Calhoun, 1990; Masson, 1979; Wright, 1968; Chen e Glad, 1991). Alguns outros fenômenos que podem originar falsas correntes diferenciais são correntes de magnetização ocorridas mediante a remoção de uma falta externa, sobreexcitação do transformador e saturação dos TCs.

A correta distinção da corrente de magnetização que surge durante a energização, de faltas internas utilizando RNAs é um dos principais propósitos desse trabalho.

\section{A SATURAÇÃO DOS TCS}

Conforme os Sistemas Elétricos de Potência crescem em tamanho e complexidade, apresentando aumento nos níveis das correntes de falta, os relés de proteção e os equipamentos de medição passam a desempenhar um papel cada vez mais importante. Neste aspecto, os TCs são empregados para fornecer uma redução das correntes primárias, bem como permitir isolação galvânica entre o sistema elétrico e os equipamentos conectados secundário do TC, incluindo os relés de proteção. Portanto, TCs são construídos para suportar correntes de falta e outros fenômenos por poucos segundos, os quais podem alcançar valores de até 50 vezes a magnitude da corrente de carga (Horowitz e Phadke, 1995).

As correntes fornecidas pelo lado secundário dos transdutores devem ser reproduções fiéis das correspondentes correntes do seu lado primário. Embora os dispositivos modernos atendam a essa condição satisfatoriamente bem, na maior parte dos casos, o projeto de proteção necessita levar em consideração o correto ajuste dos transdutores, bem como os erros de transformação eventualmente introduzidos pelos TCs, de forma que o desempenho dos relés de proteção na presença destes erros possa ser avaliado. Quando faltas ocorrem, além dos valores de corrente atingirem níveis elevados, podem conter substanciais parcelas do componente $\mathrm{CC}$ e, ainda, pode existir fluxo remanescente no núcleo do TC. Todos esses fatores podem levar à saturação do núcleo do transformador de corrente e produzir significante distorção na forma de onda secundária de corrente do dispositivo (Horowitz e Phadke, 1995). Nesse caso, a corrente secundária do TC pode não representar exatamente sua corrente primária. Portanto, relés que dependem dessa corrente para a tomada de decisão podem facilmente operar incorretamente durante esse período, afetando a eficiência da proteção.

A Figura 3 mostra um caso de falta interna simulada onde a saturação não foi representada pelo fato de um TC ideal ter sido considerado. A Figura 4 mostra o mesmo caso onde as distorções causadas pela saturação podem ser claramente observadas. Nota-se a diferença nas formas de onda dos sinais para a mesma situação analisada. Os sinais foram simulados no programa ATP.

Alguns métodos são utilizados para evitar a saturação, porém algumas das soluções podem afetar o custo de tal equipamento. O uso de RNAs surge como uma alternativa para a correção de sinais distorcidos pela saturação dos TCs (Yu et alii, 1999; Yu et alii, 2001; Kang et alii, 1997).

Neste trabalho, um método alternativo para a correção de sinais distorcidos pela saturação dos TCs foi elaborado. Para tal fim, um sistema baseado em Redes Neurais Artificiais Recorrentes foi implementado para corrigir os sinais distorcidos.

Embora muitos trabalhos abordem o problema da saturação de TCs (Yu et alii, 1999; Yu et alii, 2001; Kang et alii, 1996; Kang et alii, 1997; Jamali e Radji, 1997; Sidhu et alii, 1999; Cummins et alii, 2000), os métodos disponíveis apresentam 


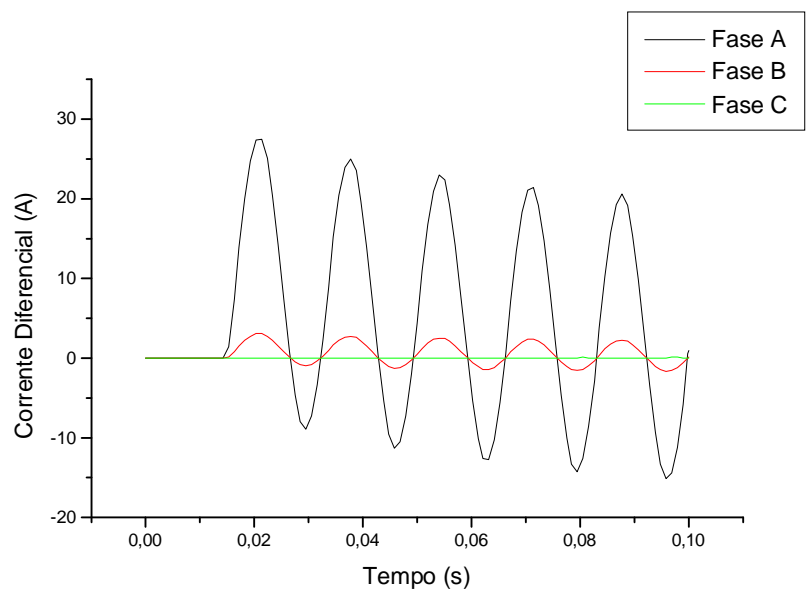

Figura 3: Caso de falta interna sem a saturação do TC

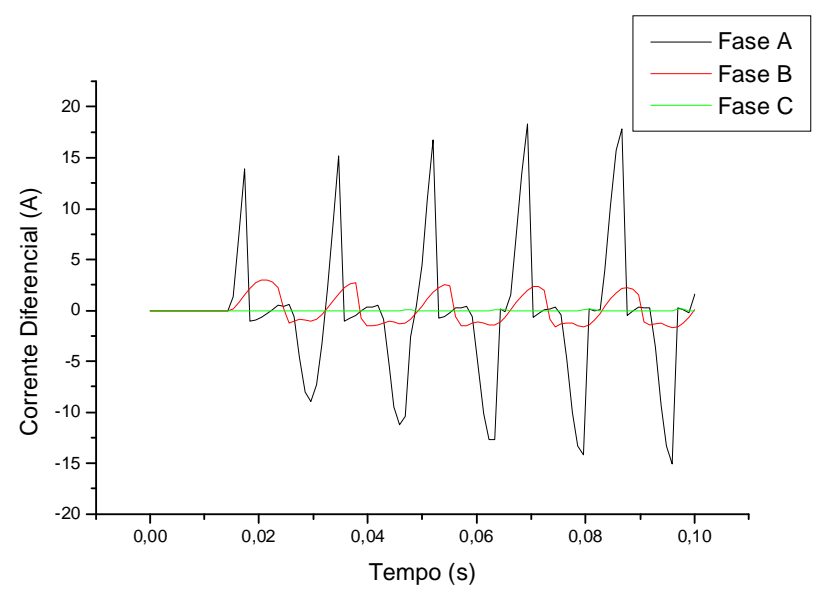

Figura 4: Caso de falta interna com a saturação do TC

certas limitações, tais como as modelagens limitadas dos TCs e a não consideração de certas situações que causam distorção nos sinais oriundos dos transdutores.

Uma maneira de evitar tal problema é aumentar o tamanho do núcleo do TC. Pode-se ainda usar um material construtivo do núcleo que suporte grandes densidades de fluxo. Ambos os métodos podem afetar o custo e a facilidade de aplicação do transformador.

Têm surgido algumas propostas para reduzir a seção transversal requerida do núcleo do TC (Bozoki e Calhoun, 1990; Masson, 1979) sem afetar a habilidade dos dispositivos em lidar com grandes trocas de fluxo.

A maioria dos relés de proteção toma decisões baseadas no valor RMS da corrente de falta. Se o sinal fornecido pelo TC é distorcido pela saturação, o valor eficaz percebido poderá ser muito menor que o valor da corrente de falta presente.
Isto pode causar atraso na operação de trip do relé. Quando a saturação do TC é maior, poderá até mesmo evitar a operação de desligamento. Esse tipo de problema tem sido largamente discutido na literatura relativa a vários tipos de esquemas de proteção (Wright, 1968; Chen e Glad, 1991; Kezunovic et alii, 1993; Barnett et alii, 1993; Glinkowski e Esztergalyos, 1996).

Em Conrad et alii (1984), um método para correção das correntes secundárias distorcidas dos TCs foi apresentado assumindo-se que a corrente de magnetização é fornecida explicitamente no cálculo. Porém, como essa corrente não pode normalmente ser fornecida para todos os casos, dificuldades práticas podem surgir.

Se a característica da saturação do TC é conhecida, uma estimativa da corrente de entrada na situação onde o TC satura pode ser encontrada, assumindo-se carga resistiva (Barnett et alii, 1993). A maioria das correntes de falta não terá a característica simétrica necessária para essa estimativa simplificada.

Alguns trabalhos têm sido realizados na tentativa de reconstrução dos sinais distorcidos pelos TCs utilizando Redes Neurais Artificiais.

A fim de lidar com diferentes faixas de correntes de falta, uma técnica que emprega dois conjuntos de coeficientes para as RNAs foi usada em (Yu et alii, 1999).

Em Cummins et alii (2000), redes neurais artificiais foram treinadas para obter a função de transferência inversa dos TCs toroidais e estimar as correntes secundárias distorcidas pela saturação dos mesmos. A velocidade e a precisão da RNA obtida mostraram-se razoáveis em aplicações em tempo real.

\section{REDES NEURAIS ARTIFICIAIS}

A rede de neurônios artificiais foi inspirada no que o homem conhece sobre o cérebro humano. O mesmo possui as características de robustez, tolerância a falhas, capacidade de ajuste a novos ambientes pelo treinamento, potencial para processar informações ruidosas, além de alta velocidade de processamento (McCulloch e Pitts, 1943).

Um modelo de neurônio artificial binário com limiar unitário foi proposto inicialmente, no qual as entradas do neurônio são ponderadas pelos seus pesos com as saídas da célula anterior ou com os sinais de entrada da rede e que possui estado de ativação definido unicamente por uma função degrau. Os pesos definem a força da sinapse conectando os neurônios.

Mais tarde, implementou-se um modelo de RNA que consiste de um conjunto de células conectadas e com uma regra 
de propagação. Cada neurônio recebe suas entradas com os pesos associados, vindas de outros neurônios ou de estímulo externo. A função de ativação é usualmente um somatório agindo nas entradas da rede. Os valores bias são adicionados ao somatório das entradas com pesos, sendo esclarecidos na próxima seção. O estado de ativação do neurônio é determinado pela função de saída ou de transferência e geralmente é uma função sigmoidal. Uma regra de propagação consiste das funções de ativação e de transferência (saída). Esta saída do neurônio trafega pelas sinapses até a próxima célula. Portanto, uma RNA é formada por um conjunto de neurônios dispostos em uma ou mais camadas, conectados por ligações de pesos variados (SNNS User Manual, 1995).

A rede feedforward tem a propagação do sinal para frente, entre células de camadas adjacentes, até a última camada (saída) (Carvalho et alii, 2000). Na fase de treinamento da rede, há a determinação e a correção dos pesos e dos bias, para que a mesma responda de uma forma desejada. O objetivo deste algoritmo de treinamento é minimizar o erro global, ou seja, minimizar a distância quadrática entre as saídas desejadas e calculadas. A descrição do algoritmo de treinamento Backpropagation mostra que RNAs de múltiplas camadas são capazes de solucionar problemas complexos ( $\mathrm{Ru}-$ melhart et alii, 1986).

A estrutura de processamento paralelo de informação permite a inclusão de conhecimento hábil no processo, a detecção e a classificação de sinais. A característica da RNA é, então, considerar o conhecimento adquirido durante o treinamento e responder a novos dados de entrada da maneira mais apropriada, concluindo assim, uma generalização do problema. A arquitetura de rede neural é definida então pelo esquema de conexões e pesos correspondentes. O processo de aprendizagem envolve a mudança nos valores dos pesos, sendo esse o aspecto mais explorado no estudo de Redes Neurais Artificiais.

\subsection{Redes Neurais Recorrentes}

Muitos algoritmos de treinamento das RNAs não são capazes de implementar mapeamentos dinâmicos, como por exemplo o algoritmo de retropropagação simples, que pode apenas aprender mapeamentos estáticos. Um artifício utilizado para processamento temporal utilizando estas redes envolve o uso de janelas de tempo, onde a entrada da rede utiliza trechos dos dados temporais como se eles formassem um padrão estático.

Entretanto, esta solução não é a mais indicada para o processamento temporal. A principal questão, portanto, é como estender a estrutura das redes MLP - Multi Layer Perceptron - para que assumam um comportamento que varie com o tempo, sendo assim capaz de tratar sinais temporais (Car- valho et alii, 2000).

O tempo pode ser representado pelo efeito que acarreta no processamento de sinais, que significa oferecer características dinâmicas ao mapeamento realizado pela rede, tornandoa sensível a sinais que variem com o tempo. Para uma RNA ser considerada dinâmica, é preciso que possua memória (Elman, 1990).

Existem basicamente duas maneiras de prover memória a uma RNA. A primeira seria introduzindo atraso no tempo, como as técnicas TDNN - Time Delay Neural Network, e FIR Multilayer Perceptron. A segunda maneira e que foi abordada neste trabalho, seria a utilização de redes neurais recorrentes, tais como Backpropagation Through Time, RealTime Recurrent Learning, Cascate Correlation recorrente, redes de Elman e redes de Jordan (Carvalho et alii, 2000).

Pode-se definir redes recorrentes como aquelas que possuem conexões de realimentação que proporcionam comportamento dinâmico. Há dois tipos de redes recorrentes: aquelas em que o padrão de entrada é fixo e a saída caminha, dinamicamente, para um estado estável e aquelas em que ambas, entrada e saída, variam com o tempo, sendo estas últimas mais gerais e utilizadas com maior freqüência.

Há muitas variações de arquiteturas de redes recorrentes, sendo que algumas delas permitem o uso de algoritmos de treinamento mais simples ou adaptados a uma tarefa particular. Duas maneiras que podem ser usadas para treinar uma rede recorrente, e que não envolvem o uso de aproximações na computação dos gradientes são Backpropagation Through Time e redes recorrentes de tempo real.

\subsection{Redes de Elman}

Elman introduziu memória às RNAs (Elman, 1990). Nas redes de Elman, além das unidades de entrada, intermediárias e de saída, há também unidades de contexto, como nas redes parcialmente recorrentes em geral. As unidades de entrada e saída interagem com o ambiente externo, enquanto as unidades intermediárias e de contexto não o fazem. As unidades de entrada são apenas unidades de armazenamento (buffer) que passam os sinais sem modificá-los. As unidades de saída são unidades lineares que somam os sinais que recebem. As unidades intermediárias podem ter funções de ativação lineares ou não lineares, e as unidades de contexto são usadas apenas para memorizar as ativações anteriores das unidades intermediárias e podem ser consideradas como atraso no tempo em um passo.

As conexões feedforward são modificáveis e as conexões recorrentes são fixas, motivo pelo qual a rede de Elman é apenas parcialmente recorrente. 


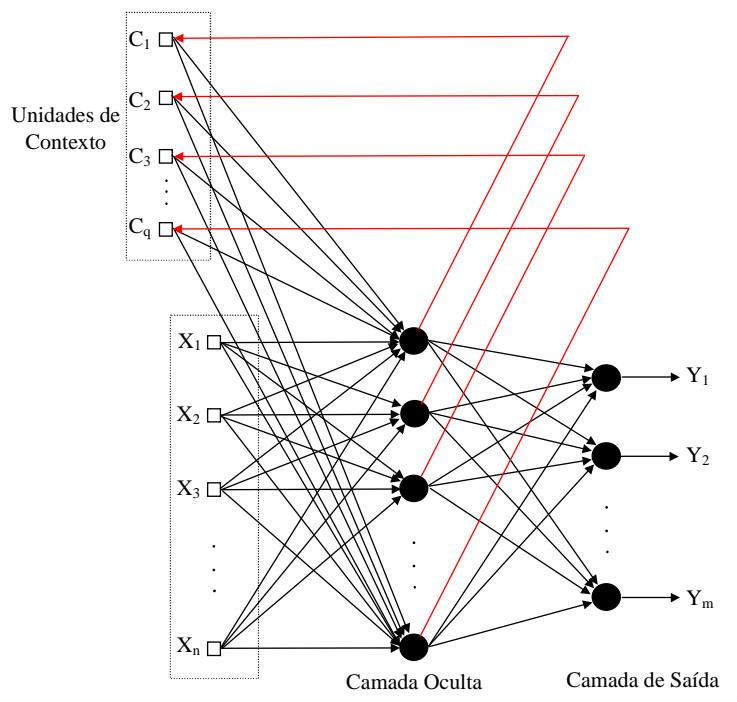

Figura 5: Rede neural de Elman

A Figura 5 ilustra a operação de uma rede de Elman.

Em um intervalo de tempo específico k, as ativações das unidades intermediárias (em k-1) e as entradas correntes (em k) são utilizadas como entradas da rede. Em um primeiro estágio, feedforward, estas entradas são propagadas para frente a fim de produzir as saídas. Posteriormente, a rede é treinada com o algoritmo de aprendizagem de retropropagação padrão. Após este passo de treinamento, as ativações das unidades intermediárias no tempo k são reintroduzidas através das ligações recorrentes nas unidades de contexto, sendo salvas nestas unidades para o próximo passo do treinamento $(\mathrm{k}+1)$.

No início do treinamento, as ativações das unidades intermediárias são desconhecidas e, geralmente, são inicializadas para a metade do valor máximo que as unidades intermediárias podem ter.

Na rede de Jordan (Jordan, 1986), a saída da rede é copiada para a unidade de contexto. Adicionalmente, as unidades de contexto são localmente recorrentes. A grande diferença em termos de topologia entre as duas redes é que a recorrência na rede de Elman é feita da camada oculta para as entradas, enquanto que na rede de Jordan a recorrência é feita das saídas para as entradas.

\section{O SISTEMA ELÉTRICO SIMULADO}

A Figura 6 mostra a representação do sistema elétrico (Coury et alii, 1998) simulado pelo programa ATP com o intuito de geração do banco de dados utilizado no treinamento e testes das RNAs. O sistema é composto de um equivalente de geração de $138 \mathrm{kV}$ e $30 \mathrm{MVA}$, um transformador de 138/13,8

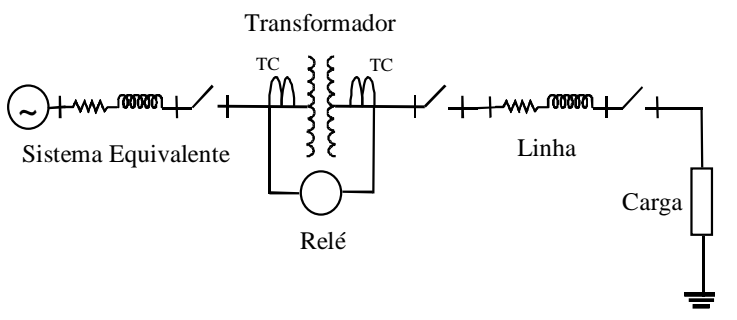

Figura 6: Sistema elétrico simulado

$\mathrm{kV}$ e 25 MVA, uma linha de transmissão de $5 \mathrm{~km}$ seguida de uma carga de 10 MVA e fator de potência 0,92 indutivo.

O transformador trifásico é conectado em configuração delta/estrela e seu modelo no EMTP-ATP foi implementado utilizando-se três transformadores monofásicos.

Um segundo transformador foi modelado para fins comparativos e uma melhor validação do trabalho. Tal transformador é idêntico ao anterior, porém com potência de 6,25 MVA e 69:13,8 kV e foi empregado na fase final do trabalho, onde foram testados os algoritmos propostos.

O número total de 2595 casos foi simulado para cada transformador usando janelas móveis de dados com três amostras, totalizando 7,785 padrões (108 casos de energização, 216 casos de faltas internas espira-terra, 216 casos de faltas internas entre espiras em ambos os lados do transformador, 162 casos de faltas externas ao transformador, 81 casos de faltas ocorrendo entre o transformador e o TC secundário, 72 casos de energização com a presença de falta interna, 45 casos de sobreexcitação do transformador, 15 casos de energização de bancos de capacitores, 54 casos de rejeição de carga, 324 casos de remoção de faltas externas e 5 casos de regime permanente). Após o processo de simulação, os dados foram organizados para a utilização no estágio de treinamento da RNA, o qual é descrito na próxima seção.

\section{RECONHECIMENTO DE PADRÕES COM A SATURAÇÃO DOS TRANSFOR- MADORES DE CORRENTE}

Os casos envolvidos nesta etapa representam somente as situações que ocasionam correntes diferenciais relevantes ao relé de proteção, ou seja, as situações de energização de bancos de capacitores e de regime permanente não foram incluídas nesta etapa. Assim, construiu-se um conjunto de dados contendo um total de 7668 padrões, ou 2556 casos, considerando-se a janela de dados móveis de 3 movimentos.

\section{O Processo de Treinamento}

Após a simulação dos dados, estes foram divididos em con- 
juntos de treinamento, validação e testes. Um total de quatro amostras por fase das correntes diferenciais foram utilizadas no treinamento com freqüência amostral de $1 \mathrm{kHz}$. Uma janela móvel de dados foi então aplicada gerando três padrões para cada caso simulado pelo ATP.

As três janelas de dados móveis para a fase A são:

Primeira janela - $\mathrm{i}_{A 1}, \mathrm{i}_{A 2}, \mathrm{i}_{A 3}, \mathrm{i}_{A 4}$

Segunda janela - $\mathrm{i}_{A 2}, \mathrm{i}_{A 3}, \mathrm{i}_{A 4}, \mathrm{i}_{A 5}$

Terceira janela - $\mathrm{i}_{A 3}, \mathrm{i}_{A 4}, \mathrm{i}_{A 5}, \mathrm{i}_{A 6}$

onde $\mathrm{i}_{A 1}, \mathrm{i}_{A 2}, \mathrm{i}_{A 3}, \mathrm{i}_{A 4}, \mathrm{i}_{A 5}$ e $\mathrm{i}_{A 6}$ são os valores discretos de corrente diferencial da fase A. O movimento de uma amostra de uma janela para a próxima pode ser notado. O procedimento é similar para as fases B e C.

Nas etapas de treinamento e validação, $50 \%$ dos casos consideraram o fenômeno de saturação causado pelos transformadores de corrente. A divisão da camada de entrada das redes neurais estudadas, em função dos sinais de correntes diferenciais gerados para cada fase e submetidos à janela de dados móveis é mostrada na Figura 7.

Para a aplicação de Redes Neurais Artificiais à proteção digital de transformadores de potência convencionou-se, para as respostas das redes, que saídas menores ou iguais a 0,5 indicariam situações de bloqueio do relé e que saídas maiores que 0,5 corresponderiam a situações nas quais o sinal de trip deveria ser enviado.

Muitas topologias de redes neurais foram construídas, treinadas e testadas para esta tarefa, porém é mencionada aqui aquela que conseguiu o melhor desempenho dentre os mode-

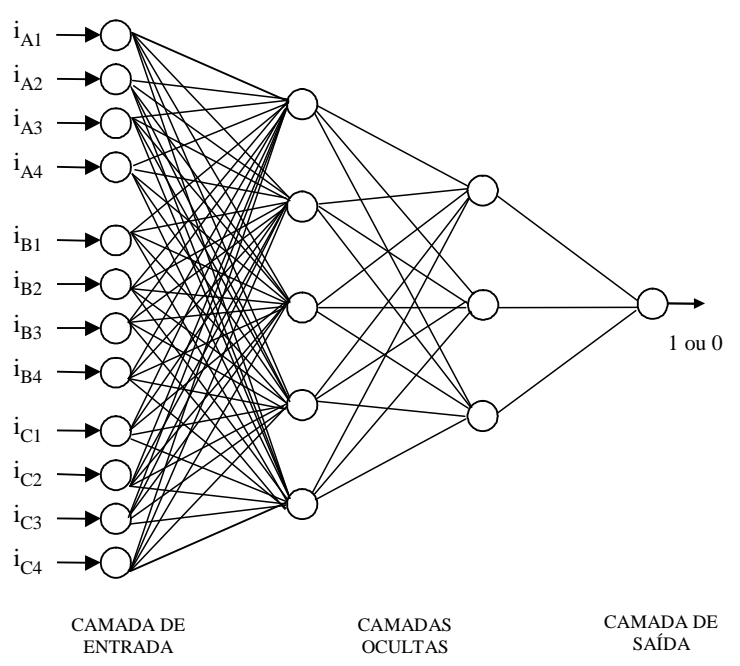

Figura 7: Representação das entradas e saídas das RNAs los testados. A melhor rede neural obtida foi do tipo MLP Backpropagation e conteve duas camadas intermediárias ou ocultas. A primeira camada constituiu-se de 8 células e a segunda, de 5 neurônios. Esta configuração foi a mais simples alcançada e capaz de fornecer $99,18 \%$ de acertos na distinção entre as situações estudadas, fornecendo 2535 respostas corretas.

\section{RNAS CORRIGINDO OS SINAIS DIS- TORCIDOS PELA SATURAÇÃO DOS TCS}

O processo de treinamento para estes casos foi idêntico àquele descrito na seção anterior, porém com a inclusão dos casos de energização de bancos de capacitores (30 casos) e de regime permanente ( 9 casos). O banco de dados total para este etapa totalizou, portanto, 2595 casos ou 7785 vetores, considerando-se a janela de dados móveis de 3 movimentos. Para os processos de treinamento e validação, $50 \%$ dos casos consideraram o fenômeno de saturação.

Usando o simulador SNNS, o método de treinamento de Elman foi executado. O estágio de treinamento convergiu em 8000 ciclos, alcançando o erro quadrático médio de 0,0005. As camadas de entrada e saída da RNA contiveram 24 neurônios cada uma (meio ciclo de dados). Nessa fase, a melhor topologia de RNA obtida foi a 24+20+24, com 24 neurônios na camada de entrada, 20 neurônios na camada oculta, 24 neurônios na camada recorrente e 24 neurônios na camada de saída, a qual reconstruiu o sinal de entrada.

\section{Resultados Obtidos}

Um total de 2595 padrões foi empregado para testar as arquiteturas empregadas. Esses dados não foram usados na etapa de treinamento.

A RNA de Elman obteve uma precisão de $98,5 \%$ na tarefa de reconstrução de sinais distorcidos. O emprego das RNAs recorrentes nesta tarefa mostrou-se muito eficiente através dos testes realizados, tornando os sinais corrigidos muito próximos das situações desejadas. Esta é uma contribuição significativa com relação à proteção do transformador que será evidenciada na eficiência de um dos algoritmos mostrados nas seções seguintes. Deve também ser ressaltado o ganho com relação à oscilografia associada ao equipamento. Os resultados referentes a esta aplicação foram mostrados em (Segatto e Coury, 2006).

\section{OS ALGORITMOS PROPOSTOS}

O algoritmo da Figura 8 ilustra o princípio lógico proposto para o relé diferencial, considerando o emprego da ferramenta baseada em Redes Neurais Artificiais em substitui- 


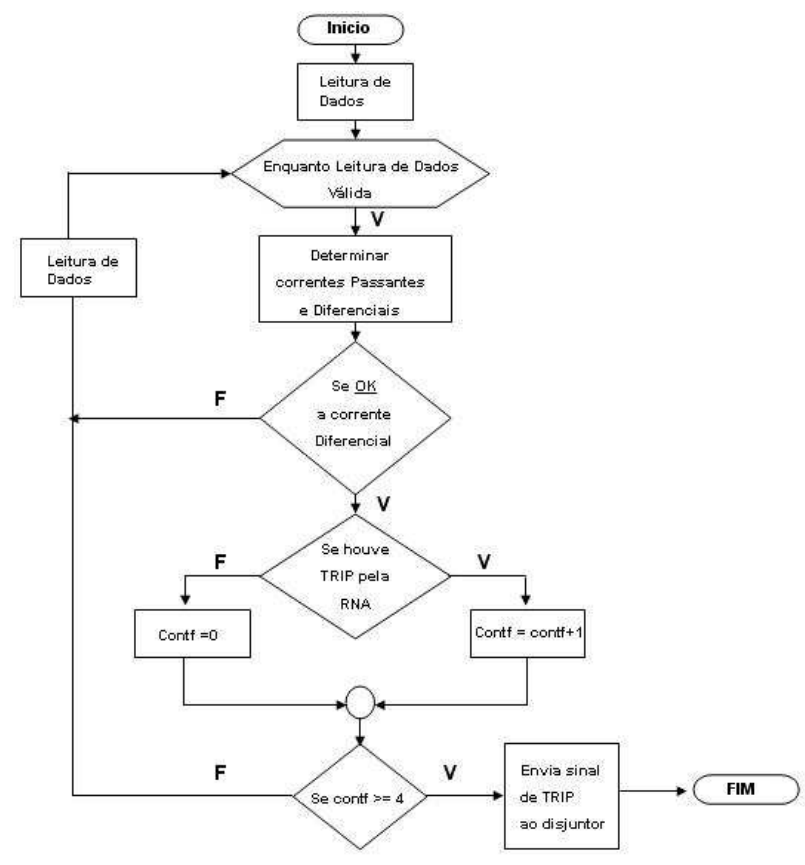

Figura 8: Primeiro algoritmo proposto

ção ao procedimento de extração de componentes harmônicos tradicional. Neste algoritmo, após a chegada dos sinais ao processador, são calculadas as correntes passantes e diferenciais. Após esta etapa, o relé verifica a ocorrência de correntes diferenciais de valores consideráveis no sistema, analisando a área de operação do dispositivo em função da característica diferencial escolhida, conforme a inclinação ajustada para a curva de restrição percentual do relé. Se a presença de correntes diferenciais não for acusada pelo dispositivo, o algoritmo retorna ao passo inicial. Caso contrário, o algoritmo realiza a verificação da condição de abertura ou bloqueio do disjuntor através da rede neural artificial. Tal ferramenta substitui então, no algoritmo proposto, a lógica convencional de restrição harmônica baseada na decomposição por Fourier. Se confirmada a condição de acionamento do relé, o algoritmo faz o acréscimo na contagem de sinais de falta e verifica o valor limite para que o contador envie o sinal de desligamento ao disjuntor correspondente. Não havendo a presença de um sinal característico de uma situação de acionamento do disjuntor, o processamento volta à sua etapa inicial.

O algoritmo de proteção mostrado envolve a metodologia proposta na qual os sinais de entrada incluem casos que contêm o efeito da distorção causada pela saturação dos transformadores de corrente. A ferramenta de inteligência artificial empregada realiza a distinção de situações de acionamento ou bloqueio do disjuntor, respondendo inclusive a dados saturados. A rede neural utilizada neste algoritmo será, por-

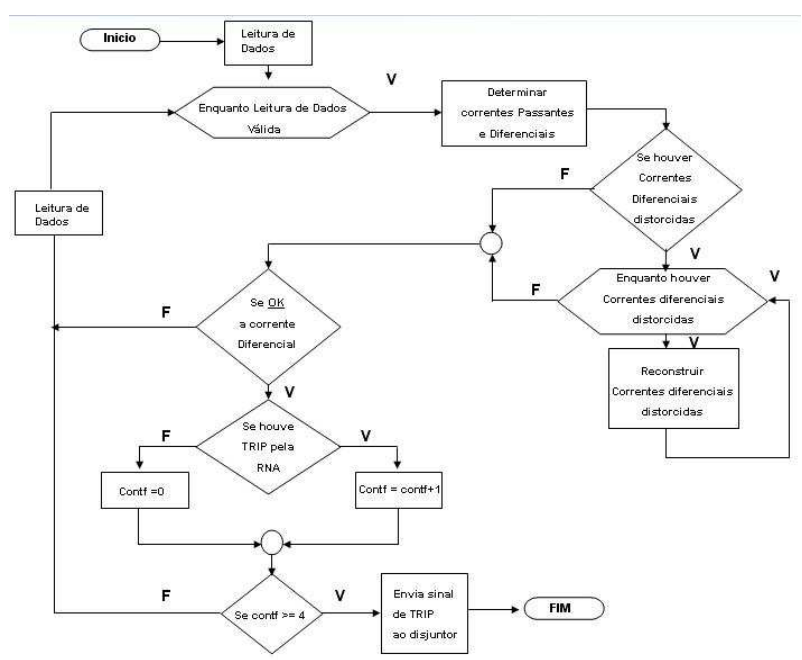

Figura 9: Segundo algoritmo proposto

tanto, treinada e testada com um banco de dados que contêm as situações de saturação mencionadas, além dos casos nos quais o fenômeno de saturação não está presente.

O esquema proposto e ilustrado na Figura 9 representa a segunda metodologia empregada neste trabalho, onde os sinais de correntes diferenciais saturados são previamente reconstruídos no início do algoritmo pelas Redes Neurais Artificiais.

O algoritmo mostrado na Figura 9 emprega, além da reconstrução de sinais saturados, o mesmo princípio lógico proposto para o relé diferencial mostrado na Fig. 8, considerando a aplicação das ferramentas baseadas em Redes Neurais Artificiais em substituição ao procedimento de extração de componentes harmônicos utilizado no algoritmo convencional. Desta forma, o primeiro módulo baseado em RNAs realiza a reconstrução dos sinais e o segundo toma a decisão de abertura ou bloqueio do disjuntor correspondente. Apesar de o algoritmo ilustrado na Figura 9 conter duas estruturas neurais implementadas, pretende-se, com essa abordagem, obter uma maior precisão nas respostas finais do algoritmo, devido à eficiência esperada pelo emprego do módulo de reconstrução de sinais distorcidos.

\section{O DESEMPENHO DO ALGORITMO CONVENCIONAL BASEADO EM DFT}

Um importante aspecto nesta etapa do trabalho é comparar a abordagem convencional com a proposta no estudo. No algoritmo convencional, os dados de entrada são as correntes diferenciais e diretas que surgem no sistema envolvido. $\mathrm{O}$ algoritmo então verifica a ocorrência de uma corrente diferencial significante capaz de sensibilizar o relé baseado na curva 
característica apresentada na Figura 2. No próximo passo, o algoritmo verifica o conteúdo harmônico do sinal, decidindo se um sinal de trip será enviado ao disjuntor.

O ajuste de inclinação da curva característica do relé depende de muitos fatores. A fim de considerar um número apropriado de possíveis valores de inclinação e evitar possíveis erros no ajuste da curva, o algoritmo foi testado levando-se em conta as inclinações de 25, 30 e 40\%. Ainda, a percentagem de componente de segunda harmônica, estimada pela Transformada de Fourier, em relação à fundamental foi variada durante os testes. Esse valor varia de acordo com o tipo de sistema de potência.

Um total de 2595 casos foi usado nos testes do algoritmo convencional, sendo que os casos testados envolveram $50 \%$ de sinais com a presença de saturação dos dispositivos transformadores de corrente. É importante novamente lembrar que o banco de dados utilizado foi elaborado a partir dos sistemas elétricos simulados com os transformadores de 25 e 6,25 MVA, o qual não foi utilizado nos estudos anteriores.

A fim de cobrir uma faixa adequada de inclinações possíveis e evitar possíveis erros de ajuste da curva, o algoritmo foi testado levando em consideração as inclinações de 25 e $40 \%$. Variou-se também durante os testes, o conteúdo do componente de segunda harmônica em relação à fundamental para os critérios de restrição em 20 e $50 \%$, pois essa relação muda de acordo com o tipo e a potência do sistema e com as alterações às quais o mesmo fica submetido.

A Tabela 1 mostra os melhores resultados obtidos pela simulação do algoritmo usando a lógica diferencial convencional para o transformador de 25 MVA. São ilustrados os resultados para uma curva característica do relé com $25 \%$ de inclinação e o conteúdo do componente de segunda harmônica em relação a fundamental sendo ajustado em $20 \%$.

A Tabela 2 mostra os melhores resultados obtidos pela simulação do algoritmo diferencial convencional para o transformador de 6,25 MVA. São ilustrados os resultados para uma curva característica com $25 \%$ de inclinação. O conteúdo do componente de segunda harmônica em relação a fundamental foi ajustado em $20 \%$.

Pode-se notar que o algoritmo diferencial convencional usando a filtragem harmônica de Fourier apresentou resultados insatisfatórios em diversas situações, demonstrando imprecisão nos testes que envolvem sinais com saturação ou conteúdo harmônico atípico.
Tabela 1: Desempenho do Algoritmo Diferencial Convencional - Transformador de 25 MVA

\begin{tabular}{|c|c|c|c|}
\hline \multicolumn{4}{|c|}{$\begin{array}{l}\text { ALGORITMO CONVENCIONAL - TRANSFORMADOR DE } 25 \\
\text { MVA }\end{array}$} \\
\hline \multicolumn{4}{|c|}{ CURVA CARACTERÍSTICA COM INCLINAÇÃO DE 25\% } \\
\hline \multicolumn{4}{|c|}{$\begin{array}{l}\text { CONTEÚDO PARA RESTRIÇÃO DE } 2^{\circ} \text { HARMÔNICO } \\
\qquad I_{2} / I_{1}=20 \%\end{array}$} \\
\hline CASOS TESTADOS & $\begin{array}{l}\text { NÚMERO } \\
\text { DE } \\
\text { PADRÕES }\end{array}$ & ACERTOS & ERROS \\
\hline Energização & 216 & 137 & 79 \\
\hline Faltas Internas & 864 & 693 & 171 \\
\hline Faltas Externas & 324 & 228 & 96 \\
\hline $\begin{array}{c}\text { Faltas entre } \\
\text { Transformador e TC }\end{array}$ & 162 & 105 & 57 \\
\hline $\begin{array}{l}\text { Energização com Falta } \\
\text { Interna }\end{array}$ & 144 & 93 & 51 \\
\hline Sobre-excitação & 90 & 65 & 25 \\
\hline $\begin{array}{l}\text { Energização de Bancos } \\
\text { de Capacitores }\end{array}$ & 30 & 23 & 7 \\
\hline Rejeição de Cargas & 108 & 72 & 36 \\
\hline $\begin{array}{l}\text { Remoção de Faltas } \\
\text { Externas }\end{array}$ & 648 & 489 & 159 \\
\hline Regime Permanente & 9 & 9 & 0 \\
\hline TOTAL & 2595 & 1914 & 681 \\
\hline TOTAL $(\%)$ & 100,00 & 73,76 & 26,24 \\
\hline
\end{tabular}

\section{O DESEMPENHO DOS ALGORITMOS PROPOSTOS}

Nesta seção são analisados os desempenhos dos 2 algoritmos propostos no trabalho e ilustrados nas Figuras 8 e 9 . O banco de dados utilizado para esta tarefa foi idêntico ao empregado na análise do algoritmo tradicional baseado em DFT descrito na seção anterior, ou seja, foram utilizados 2,595 padrões, sendo que os casos testados envolveram $50 \%$ de sinais com a presença de saturação dos dispositivos transformadores de corrente. É importante novamente lembrar que o banco de dados utilizado foi elaborado a partir dos sistemas elétricos simulados com os transformadores de 25 e 6,25 MVA.

A Tabela 3 ilustra o desempenho do primeiro algoritmo proposto, que utiliza somente o reconhecimento de padrões realizado através da RNA MLP Backpropagation, para o transformador de 25 MVA. A Tabela 4 mostra o desempenho do mesmo algoritmo para o transformador de 6,25 MVA.

A Tabela 5 ilustra o desempenho do segundo algoritmo proposto, que utiliza o reconhecimento de padrões (rede MLP) aliado à reconstrução de sinais distorcidos (rede recorrente de 
Tabela 2: Desempenho do Algoritmo Diferencial Convencional - Transformador de 6,25 MVA

\begin{tabular}{|c|c|c|c|}
\hline \multicolumn{3}{|c|}{ ALGORITMO CONVENCIONAL - TRANSFORMADOR DE 6,25 } \\
MVA \\
\hline \multicolumn{3}{|c|}{ CURVA CARACTERÍSTICA COM INCLINAÇÃO DE 25\% } \\
\hline \multicolumn{3}{|c|}{ CONTEÚDO PARA RESTRIÇÃO DE $2^{\circ}$ HARMÔNICO } \\
\hline I $I_{1}=20 \%$ \\
CASOS TESTADOS & $\begin{array}{c}\text { NÚMERO } \\
\text { DE } \\
\text { PADRÕES }\end{array}$ & ACERTOS & ERROS \\
\hline Energização & 216 & 132 & 84 \\
Faltas Internas & 864 & 672 & 192 \\
Faltas Externas & 324 & 222 & 102 \\
$\begin{array}{c}\text { Faltas entre } \\
\text { Transformador e TC } \\
\text { Energização com Falta } \\
\text { Interna }\end{array}$ & 162 & 102 & 60 \\
$\begin{array}{c}\text { Sobre-excitação } \\
\text { Energização de Bancos } \\
\text { de Capacitores }\end{array}$ & 90 & 91 & 53 \\
$\begin{array}{c}\text { Rejeição de Cargas } \\
\text { Remoção de Faltas } \\
\text { Externas }\end{array}$ & 108 & 70 & 25 \\
$\begin{array}{c}\text { Regime Permanente } \\
\text { TOTAL }\end{array}$ & 948 & 473 & 175 \\
\hline TOTAL (\%) & 100,00 & 71,60 & 28,40 \\
\hline
\end{tabular}

Elman) para o transformador de 25 MVA. A Tabela 6 mostra o desempenho do mesmo algoritmo para o transformador de 6,25 MVA.

Pode-se notar que, de maneira análoga ao primeiro algoritmo proposto, apesar de a ferramenta ter sido testada também com dados gerados por um sistema elétrico de configuração diferente daquele utilizado para obter os dados de treinamento das RNAs em questão, o desempenho do algoritmo mostrou-se muito elevado para ambos os transformadores, como pode ser observado pela alta precisão alcançada.

Ressalta-se que este segundo algoritmo proposto supera, em termos de precisão, o primeiro algoritmo, o qual utilizou apenas uma estrutura neural tipo MLP a fim de realizar o reconhecimento de padrões necessário para a tomada de decisão do relé.

Conclui-se, portanto, que a ferramenta de inteligência artificial que envolveu o módulo adicional de reconstrução de sinais alcançou elevada precisão mesmo quando testada com dados gerados por um segundo sistema elétrico, o que vem atestar o alto desempenho e versatilidade atingidos por essa
Tabela 3: Desempenho do Primeiro Algoritmo Proposto Transformador de 25 MVA

\begin{tabular}{|c|c|c|c|}
\hline \multicolumn{4}{|c|}{ ALGORITMO 1 - TRANSFORMADOR DE 25 MVA } \\
\hline CASOS TESTADOS & $\begin{array}{l}\text { NÚMERO } \\
\text { DE } \\
\text { PADRÕES }\end{array}$ & ACERTOS & ERROS \\
\hline Energização & 216 & 208 & 8 \\
\hline Faltas Internas & 864 & 855 & 9 \\
\hline Faltas Externas & 324 & 321 & 3 \\
\hline $\begin{array}{c}\text { Faltas entre } \\
\text { Transformador e TC }\end{array}$ & 162 & 160 & 2 \\
\hline $\begin{array}{l}\text { Energização com Falta } \\
\text { Interna }\end{array}$ & 144 & 143 & 1 \\
\hline Sobre-excitação & 90 & 89 & 1 \\
\hline $\begin{array}{l}\text { Energização de Bancos } \\
\text { de Capacitores }\end{array}$ & 30 & 30 & 0 \\
\hline Rejeição de Cargas & 108 & 105 & 3 \\
\hline $\begin{array}{c}\text { Remoção de Faltas } \\
\text { Externas }\end{array}$ & 648 & 640 & 8 \\
\hline Regime Permanente & 9 & 9 & 0 \\
\hline TOTAL & 2595 & 2560 & 35 \\
\hline TOTAL $(\%)$ & 100,00 & 98,65 & 1,35 \\
\hline
\end{tabular}

Tabela 4: Desempenho do Primeiro Algoritmo Proposto Transformador de 6,25 MVA

\begin{tabular}{|c|c|c|c|}
\hline \multicolumn{4}{|c|}{ ALGORITMO 1 - TRANSFORMADOR DE 6,25 MVA } \\
\hline CASOS TESTADOS & $\begin{array}{l}\text { NÚMERO } \\
\text { DE } \\
\text { PADRÕES }\end{array}$ & ACERTOS & ERROS \\
\hline Energização & 216 & 206 & 10 \\
\hline Faltas Internas & 864 & 850 & 14 \\
\hline Faltas Externas & 324 & 318 & 6 \\
\hline $\begin{array}{c}\text { Faltas entre } \\
\text { Transformador e TC }\end{array}$ & 162 & 158 & 4 \\
\hline $\begin{array}{l}\text { Energização com Falta } \\
\text { Interna }\end{array}$ & 144 & 141 & 3 \\
\hline Sobre-excitação & 90 & 88 & 2 \\
\hline $\begin{array}{l}\text { Energização de Bancos } \\
\text { de Capacitores }\end{array}$ & 30 & 29 & 1 \\
\hline Rejeição de Cargas & 108 & 103 & 5 \\
\hline $\begin{array}{c}\text { Remoção de Faltas } \\
\text { Externas }\end{array}$ & 648 & 636 & 12 \\
\hline Regime Permanente & 9 & 9 & 0 \\
\hline TOTAL & 2595 & 2538 & 57 \\
\hline TOTAL (\%) & 100,00 & 97,80 & 2,20 \\
\hline
\end{tabular}


Tabela 5: Desempenho do Segundo Algoritmo Proposto Transformador de 25 MVA

\begin{tabular}{|c|c|c|c|}
\hline \multicolumn{4}{|c|}{ ALGORITMO 2 TRANSFORMADOR DE 25 MVA } \\
\hline CASOS TESTADOS & $\begin{array}{c}\text { NÚMERO } \\
\text { DE } \\
\text { PADRÕES }\end{array}$ & ACERTOS & ERROS \\
\hline Energização & 216 & 216 & 0 \\
Faltas Internas & 864 & 863 & 1 \\
$\begin{array}{c}\text { Faltas Externas } \\
\text { Faltas entre }\end{array}$ & 324 & 324 & 0 \\
Transformador e TC & 162 & 162 & 0 \\
Energização com Falta \\
Interna
\end{tabular}

Tabela 6: Desempenho do Segundo Algoritmo Proposto Transformador de 6,25 MVA

\begin{tabular}{|c|c|c|c|}
\hline \multicolumn{4}{|c|}{ ALGORITMO 2 - TRANSFORMADOR DE 6,25 MVA } \\
\hline CASOS TESTADOS & $\begin{array}{c}\text { NÚMERO } \\
\text { DE } \\
\text { PADRÕES }\end{array}$ & ACERTOS & ERROS \\
\hline Energização & 216 & 213 & 3 \\
Faltas Internas & 864 & 859 & 5 \\
$\begin{array}{c}\text { Faltas Externas } \\
\text { Faltas entre }\end{array}$ & 324 & 322 & 2 \\
Transformador e TC & 162 & 161 & 1 \\
Energização com Falta \\
Interna
\end{tabular}

ferramenta.

\section{COMPARAÇÃO ENTRE OS ALGORIT- MOS}

A comparação entre as metodologias estudadas neste trabalho foi realizada nesta seção com o intuito de fornecer condições de análise global dos algoritmos propostos e convencionais de proteção de transformadores de potência, permitindo assim um estudo sobre os aspectos mais relevantes das ferramentas em questão.

Desta forma, são comparados os níveis de precisão e os tempos de resposta de cada um dos seguintes algoritmos, em ambos os transformadores estudados:

1. Algoritmo convencional de proteção diferencial do transformador utilizando filtragem harmônica de Fourier;

2. Algoritmo com a utilização direta das melhores RNAs tipo MLP treinadas com a inclusão de dados saturados;

3. Algoritmo envolvendo a reconstrução de sinais saturados aliado ao reconhecimento de padrões.

Na Tabela 7 e Tabela 8, os desempenhos dos melhores algoritmos obtidos no trabalho foram destacados, para ambos os transformadores.

Pode-se afirmar inicialmente que as duas abordagens propostas envolvendo a aplicação de redes neurais artificiais aos algoritmos de proteção apresentaram desempenhos muito superiores se comparadas aos algoritmos convencionais. Tal superioridade se mostrou em relação à precisão alcançada e à velocidade de resposta dos mesmos.

Nota-se que, enquanto nas aplicações convencionais o índice de precisão permaneceu em $71,60 \%$, nas metodologias que fazem uso das RNAs este índice passou para, no mínimo,

Tabela 7: Comparação entre os Algoritmos de Proteção Propostos e Convencional para o Transformador de 25 MVA

\begin{tabular}{|c|c|c|c|}
\hline \multirow{3}{*}{ ALGORITMO } & \multirow{3}{|c|}{ TRANSFORMADOR DE 25 MVA } \\
\cline { 3 - 4 } & PRECISÃO (\%) & $\begin{array}{c}\text { Amostras } \\
\text { por Ciclo } \\
\text { Utilizadas }\end{array}$ & $\begin{array}{c}\text { Tempo de } \\
\text { Resposta } \\
(\mathrm{ms})\end{array}$ \\
\hline 1 & 73,76 & 16 & 16,67 \\
2 & 98,65 & 4 & 4,17 \\
3 & 99,92 & 8 & 8,33 \\
\hline
\end{tabular}


Tabela 8: Comparação entre os Algoritmos de Proteção Propostos e Convencional para o Transformador de 6,25 MVA

\begin{tabular}{|c|c|c|c|}
\hline \multirow{2}{*}{ ALGORITMO } & TRANSFORMADOR DE 6,25 MVA \\
\cline { 3 - 4 } & PRECISÃO (\%) & $\begin{array}{c}\text { VELLOCIDADE } \\
\text { por Ciclo } \\
\text { Utilizadas }\end{array}$ & $\begin{array}{c}\text { Tempo de } \\
\text { Resposta } \\
(\mathrm{ms})\end{array}$ \\
\hline 1 & 71,60 & 16 & 16,67 \\
2 & 97,80 & 4 & 4,17 \\
3 & 99,31 & 8 & 8,33 \\
\hline
\end{tabular}

$97,80 \%$ para os testes efetuados nos algoritmos do transformador de 6,25 MVA.

Além disso, a resposta dos algoritmos baseados em redes neurais é significativamente mais rápida, pois a abordagem convencional de Fourier necessita de, pelo menos, um ciclo de dados (16 amostras, neste caso), para responder a um sinal de entrada, considerando-se uma freqüência amostral de $1 \mathrm{kHz}$.

A decisão sobre qual das duas metodologias propostas constitui a melhor alternativa ao método convencional é extremamente criteriosa. O método que forneceu a resposta em menor tempo representa uma poderosa ferramenta em termos de proteção elétrica. Por sua vez, o segundo algoritmo proposto atingiu um índice de precisão ainda maior, embora necessite de um tempo de análise maior ( 8 amostras por ciclo). Este algoritmo, além de desempenhar as funções de proteção de transformadores de potência, possui a versatilidade de aplicações em funções de oscilografia do sistema, pelo fato de realizar a reconstrução de sinais saturados de correntes diferenciais.

É importante ressaltar que, independentemente da escolha a ser feita em função da aplicabilidade dos algoritmos propostos discutidos, estes mantêm grande vantagem sobre as metodologias convencionais relativas a esse tipo de proteção, no que diz respeito à precisão e ao tempo de resposta necessária aos algoritmos.

\section{CONCLUSÕES}

Este trabalho apresentou o desenvolvimento e a implantação de um sistema completo de proteção diferencial de transformadores de potência aplicando-se as técnicas de Redes Neurais Artificiais. Os métodos propostos trataram o esquema de proteção diferencial como um problema de reconhecimento e reconstrução de padrões, representando uma solução alternativa aos algoritmos convencionais. O trabalho abordou dois tipos propostos de algoritmos de proteção de transformado- res de potência utilizando inteligência artificial e realizou a análise comparativa entre eles. O algoritmo convencional de proteção diferencial foi também implementado a fim de melhor avaliar as metodologias desenvolvidas.

O primeiro algoritmo proposto envolveu a utilização de redes neurais do tipo MLP, com o intuito de realizar o reconhecimento de padrões usando um banco de dados que inclui situações em que tenha ocorrido a saturação dos transformadores de corrente. As aplicações mais eficientes para esse tipo de algoritmo obtiveram índices de acertos de $98,65 \%$ e $97,80 \%$ para os transformadores de 25 MVA e 6,25 MVA, respectivamente, usando-se uma estrutura neural do tipo MLP e necessitando de um tempo de resposta de $4,17 \mathrm{~ms}$, ou seja, 1/4 de ciclo de dados.

O segundo algoritmo proposto realizou o reconhecimento de padrões com o emprego de um segundo módulo de RNAs, utilizado na reconstrução dos sinais saturados. Tal metodologia utilizou a mesma rede neural do tipo MLP anterior na distinção de padrões, porém, fez uso ainda de uma rede neural recorrente na tarefa de reconstrução dos padrões distorcidos. Nesta aplicação, o índice de respostas corretas fornecido foi de $99,92 \%$ e $99,31 \%$ para os transformadores de 25 MVA e 6,25 MVA respectivamente, valores esses superiores ao algoritmo anterior, embora a resposta seja fornecida após a computação de $1 / 2$ ciclo de dados, ou seja, 8,33 ms. Esta ferramenta disponibiliza ainda um possível recurso para a função de oscilografia do sistema, já que realiza a perfeita reconstrução das correntes diferenciais de entrada que estejam afetadas pelo fenômeno de saturação dos TCs.

O algoritmo convencional de proteção diferencial foi avaliado através de testes realizados com o mesmo banco de dados utilizado nos testes dos algoritmos anteriores, para ambos os transformadores. Variaram-se, ainda, os valores de inclinação da curva característica do relé diferencial e as percentagens de conteúdo de $2^{\circ}$ harmônico em relação ao componente fundamental, para a lógica de restrição convencional. Com isso, cobriu-se uma vasta gama de testes para esta ferramenta. Entretanto, o desempenho desse algoritmo mostrou-se insatisfatório, apresentando índices gerais de acertos de apenas $73,76 \%$ e $71,60 \%$ para os transformadores de 25 MVA e 6,25 MVA, respectivamente, considerandose o mesmo nível de saturação presente nos sinais de entrada nas implementações baseadas em redes neurais. Além disso, é preciso considerar que a metodologia baseada na decomposição harmônica de Fourier necessita de, no mínimo, um ciclo de dados a fim de fornecer a resposta a um sinal de entrada, tempo este muito superior ao obtido pelos algoritmos anteriores.

Importantes constatações foram realizadas com ênfase na eficiência dos algoritmos propostos, considerando os aspectos 
mais relevantes para suas aplicabilidades, como precisão, velocidade e estrutura das ferramentas.

Destaca-se que os resultados encorajadores, conseguidos pela implementação dos algoritmos baseados em inteligência artificial, foram gerados pelos testes realizados com dados advindos, inclusive, de um sistema elétrico diferente daquele empregado no treinamento das Redes Neurais Artificiais, atestando, desta forma, a flexibilidade dos métodos propostos.

\section{AGRADECIMENTOS}

Os autores gostariam de agradecer o Departamento de Engenharia Elétrica da Escola de Engenharia de São Carlos Universidade de São Paulo, pelo apoio à pesquisa realizada, bem como o suporte financeiro concedido pela FAPESP Fundação de Amparo a Pesquisa do Estado de São Paulo.

\section{REFERÊNCIAS}

Alternative Transients Program Rule Book (1987), Leuven EMTP Center.

Barnett, C.W. and Chadwick, J.W. (1993). Relay Performance Considerations with Low Ratio CTs and High Fault Currents, IEEE Transactions on Power Delivery, v.8, n.3, pp. 884-887.

Bozoki, B. and Calhoun C.M. (1990). Gapped Core Transformer Characteristics and Performance, IEEE Power System Relaying Committee, IEEE Transactions PWRD, v.5, n.4, pp. 1732-1740.

Carvalho, A.C.P.L.F. ; Braga, A.P. e Ludermir, T.B. (2000), Redes Neurais Artificiais: Teorias e Aplicações, Rio de Janeiro, Livros Técnicos e Científicos, 262 p.

Chen, K.W. and Glad, S.T. (1991). Estimation of the Primary Current in a Saturated Transformer, Proceedings of the IEEE Conference on Decision and Control, pp. 116124.

Conrad, T. ; Schlabbach, J. and Speh, R. (1984). Verfahren zur Korrektur der verzerrten Sekundarstrome von Stromwandlern, Electrotechnische Zeitschrift Archiv, v.6, n.2, pp. 77-79.

Coury, D.V.; Campos, P.G. and Tavares, M.C. (1998). Modelling a Power Transformer for Investigation of Digital Protection Schemes. 8th International Conference on Harmonics and Quality of Power (ICHQP'98), pp. 489-494.

Cummins, J.C. ; Yu, D.C. and Kojovic, L.A. (2000). Simplified Artificial Neural Network Structure with the Current Transformer Saturation Detector provides a Good
Estimate of Primary Currents, 2000 Power Engineering Society Summer Meeting, v.3.

Elman, J.L. (1990). Finding Structure in Time. Cognitive Science, n.14, pp.179-211.

Glinkowski, M.T. and Esztergalyos, J. (1996). Transient Modeling of Electromechanical Relays, Part 1: Armature Type Overcurrent Relay, IEEE Transactions on Power Delivery, v.11, n.2.

Horowitz, S. H. and Phadke, A.G. (1995). Power System Relaying, Research Studies Press Ltd., 2nd ed., England.

Jamali, S. and Radji, K.M. (1997). A Digital Compensating Technique for the Saturation of Protective Current Transformers, Proceedings of Thirty Second Universities Power Engineering Conference, v.1, pp. 10-12.

Jordan, M.I. (1986). Attractor Dynamics and Parallelism in a Connectionist Sequential Machine. Proc. of the Eighth Annual Conf. of the Cognitive Science Society, pp. 531546.

Kang, Y.C. ; Kang, S.H. ; Park, J.K. ; Johns, A.T. and Aggarwal, R.K. (1997). Algorithm for Compensating Secondary Currents of Current Transformers, IEEE Transactions on Power Delivery, v.12, n.1, p. 116-124.

Kang, Y.C. ; Kang, S.H. ; Park, J.K. ; Johns, A.T. and Aggarwal, R.K. (1996). Development in Hardware Implementation of a Compensating Algorithm for the Secondary Current of Current Transformers, IEEE Proceedings in Electric Power Applications, v.143, n.1, pp. 41-49.

Kezunovic, M. ; Kojovic, L.A. ; Fromen, C.W. ; Sevcik, D.R. and Phillips, F. (1993). Experimental Evaluation of EMTP-based Current Transformer Models for Protective Relay Transient Study, 93 WM 041-4 PWRD, IEEE/PES Winter Meeting, New York.

Masson, L. (1979). Circuit for the Dynamic Control of Magnetic Flux in Current Transformers, IEEE Transactions PAS, v.98, n.6, pp. 1990-1995.

McCulloch, W. S. and Pitts, W. (1943). A Logical Calculus of the Ideas Immanent in Nervous Activity. Bulletin of Mathematical Biophysics, n.5, pp. 115-133.

Rumelhart, D. E., Hinton, G. E. and Williams, R.J. (1986). Learning Representations by Back-propagation Errors, Nature, n.323, pp. 533-536.

Segatto, E.C. e Coury, D.V. (2006). Redes Neurais Artificiais Recorrentes Aplicadas na Correção de Sinais Distorcidos pela Saturação de Transformadores de Corrente, SBA - Controle \& Automação, v.17, n. 4, v. 17, pp.424436. 
Sidhu, T.S. ; Gill, H.S. and Sachdev, M.S. (1999). A Power Transformer Protection Technique with Stability during Current Transformer Saturation and Ratio-mismatch Conditions, IEEE Transactions on Power Delivery, v.14, n.3, pp. 798-804.

SNNS User Manual (1995). Institute for Parallel and Distributed High Performance Systems, University of Stuttgard.

Wright, A. (1968). Current Transformers, their Transient and Steady State Performance, London: Chapman \& Hall, p. 77.

Yu, D.C. ; Cummins, J.C. ; Wang, Z. ; Yoon, H.J. and Kojovic, L.A. (2001). Correction of Current Transformer Distorted Secondary Currents due to Saturation using Artificial Neural Networks, IEEE Transactions on Power Delivery, v.16, n.2, pp.189-194.

Yu, D.C. ; Wang, Z. ; Cummins, J.C. ; Yoon, H.J. and Kojovic, L.A. (1999). Neural Network for Current Transformer Saturation Correction, Proceedings of IEEE Power Engineering Society - Transmission and Distribution Conference, v.1, pp. 441-446. 